\title{
Can a simple fingertip temperature measurement predict a cardiac event?
}

\author{
Songtao Liu · David A. Bluemke
}

Received: 29 July 2009/Accepted: 16 October 2009/Published online: 13 November 2009

(C) U.S. Government 2009

An impressive amount of evidence has suggested that vascular endothelium plays an important role in the control of vascular function and structure by the production of nitric oxide. A dysfunctional endothelium is an early marker of the development of atherosclerotic changes and can also contribute to cardiovascular events [1]. A wide range of noninvasive vascular reactivity tests have been developed to assess peripheral macrocirculation (conduit arteries) and microcirculation (resistance arteries and arterioles) non-invasively [2]. These tests have provided not only novel insights into cardiovascular pathophysiology, but also possible clinical opportunities to detect early disease, to stratify cardiovascular risk and to assess response to pharmacological interventions [3].

In a previous issue of the Journal, Ahmadi and colleagues report the result of a single-center, cross-

Editorial Comment on the article of Ahmadi et al. (2009) Int J Cardiovasc Imaging 25:725-738 (doi:10.1007/s10554-009-

9476-8).

S. Liu · D. A. Bluemke $(\bowtie)$

Radiology and Imaging Sciences, National Institutes

of Health/Clinical Center, 9000 Rockville Pike, Bldg 10/ Rm 1C355, Bethesda, MD 20892, USA

e-mail: bluemked@nih.gov

D. A. Bluemke

National Institute of Biomedical Imaging and

Bioengineering, Bethesda, MDUSA sectional trial comparing the fingertip Digital Thermal Monitoring (DTM) with CT angiography (CTA) in symptomatic elderly patients [4]. They performed DTM of both hands during 5 min of stabilization, 5 min of cuff inflation and 5 min of deflation using a high precision automatic thermometry system, and they compared the DTM measurements with the coronary artery calcium (CAC) score and CTAdiagnosed coronary artery disease (CAD). All DTM indices of vascular and neurovascular reactivity significantly decreased between normal, non-obstructive and obstructive CAD subjects. In addition, the extent of CAD increased with increasing CAC and vascular dysfunction. Multivariate logistic regression analysis demonstrated that DTM indices and CAC were independent predictors of the extent of CAD.

The results obtained in this study demonstrate a correlation between DTM and the presence and extent of CAD measured by CTA. These results are also quite similar to the inverse correlation of DTM and the myocardial perfusion defect measured by single photon emission computed tomography (SPECT) in symptomatic patients reported by the same group [5].

Atherosclerotic heart disease remains the most common cause of death and disability in the industrialized world. Improved methods are needed to predict the development of atherosclerosis and cardiac events. The field of non-invasive vascular reactivity testing for the assessment of endothelial function is interesting with respect to this need. The 
most widely used technique is flow-mediated dilatation (FMD), which functions by measuring the brachial artery diameter changes induced by an increase in shear stress [6]. FMD has shown the presence of endothelial dysfunction in patients with cardiovascular risk factors and disease [7]. Furthermore, research has demonstrated that FMD can significantly predict cardiovascular event, independently of traditional cardiovascular risk factors [6]. However, this technique is limited by some practical challenges, such as the need for highly trained operators. DTM, a non-invasive, operator-independent test, measures the reactive hyperemia caused by micro-vascular activity [8]. Although the reactive hyperemia is only partially endothelium and nitric oxide dependent, this does not limit the ability of DTM to identify groups at risk [9].

This study is limited by several factors, the most important of which is the lack of an age, and gender matched control group. Ethnicity was also not addressed in this initial study. Overall, the authors' primary aim was to compare DTM to Framingham risk score to predict CTA detected CAD. However, the Framingham score was designed to estimate 10 years risk of myocardial infarction and coronary death-not CTA identified plaque. Thus, the authors' multi-factorial regression models that link CAC (plaque) + DTM (vascular reactivity) + Framingham score (10 years event prediction) in order to predict CTA plaque are unusual constructs primarily for exploratory purposes. Finally, the cross sectional design of this research does not establish causal relationships between CAD and DTM. A multi-center longitudinal study will be needed to test the sensitivity and specificity of DTM especially in regards to brachial reactivity testing. Fortunately, DTM is noninvasive and likely inexpensive so that further investigations into its utility are likely to be undertaken.

\section{References}

1. Lerman A, Zeiher AM (2005) Endothelial function: cardiac events. Circulation 111:363-368

2. Deanfield J, Donald A, Ferri C et al (2005) Endothelial function and dysfunction. Part I: methodological issues for assessment in the different vascular beds: a statement by the Working Group on Endothelin and Endothelial Factors of the European Society of Hypertension. J Hypertens 23:7-17

3. Deanfield JE, Halcox JP, Rabelink TJ (2007) Endothelial function and dysfunction: testing and clinical relevance. Circulation 115:1285-1295

4. Ahmadi $\mathrm{N}$ et al (2009) Low fingertip temperature rebound measured by digital thermal monitoring strongly correlates with the presence and extent of coronary artery disease diagnosed by 64-slice multi-detector computed tomography. Int J Cardiovasc Imaging 25:725-738. doi:10.1007/s10554009-9476-8

5. Ahmadi N, Usman N, Shim J et al (2009) Vascular dysfunction measured by fingertip thermal monitoring is associated with the extent of myocardial perfusion defect. J Nucl Cardiol 16:431-439

6. Celermajer DS, Sorensen KE, Gooch VM et al (1992) Noninvasive detection of endothelial dysfunction in children and adults at risk of atherosclerosis. Lancet 340:1111-1115

7. Brunner H, Cockcroft JR, Deanfield J et al (2005) Endothelial function and dysfunction. Part II: Association with cardiovascular risk factors and diseases. A statement by the Working Group on Endothelins and Endothelial Factors of the European Society of Hypertension. J Hypertens 23:233246

8. Higashi Y, Sasaki S, Nakagawa K, Matsuura H, Kajiyama G, Oshima T (2001) A noninvasive measurement of reactive hyperemia that can be used to assess resistance artery endothelial function in humans. Am J Cardiol 87:121-125 (A129)

9. Ghiadoni L (2007) Change in forearm reactive hyperemia after telmisartan: an endothelial effect? Am J Hypertens 20:1312-1313 